\title{
Finasteride in hospitalized adult males with COVID-19: A risk factor for severity of the disease or an adjunct treatment: A randomized controlled clinical trial
}

\author{
Elham Zarehoseinzade ${ }^{1}$, Abbas Allami $^{1 *}$ (D) Mehrnoosh Ahmadi ${ }^{2}$, Behzad Bijani $^{1}$, Navid Mohammadi $^{3,4}$ \\ Received: 29 Sep 2020 \\ Published: 3 Mar 2021
}

\section{Abstract}

Background: There is controversy about the efficacy of 5-alpha-reductase inhibitors in COVID-19 patients. Some assumed that finasteride might be a risk factor for deterioration and others proposed it as a possible adjunct treatment for moderate to severe COVID-19 infection in the elderly.

Methods: We performed a randomized controlled clinical trial (registration ID IRCT20200505047318N1) on 80 hospitalized male patients aged $\geq 50$ years diagnosed with COVID-19 pneumonia in a tertiary hospital in Qazvin (Iran) from April to July 2020 . The patients were randomized into one of the 2 treatment groups using simple randomization. Treatment group patients underwent routine drug therapy and $5 \mathrm{mg}$ finasteride once daily for 7 days. The primary endpoint was mortality rate and length of hospital stay (LOS), and secondary endpoints were peripheral capillary oxygen saturation, respiratory rate, and inflammatory markers changes. The study protocol was approved by the medical ethics committee of Qazvin University of Medical Sciences (registration ID IR.QUMS.REC.1399.080). Data were analyzed by statistical tests and SPSS version 25 . Also, $<<0.05$ was considered to be statistically significant.

Results: We found a significant difference on $\mathrm{O}_{2}$ saturation among the 2 study groups on fifth day compared with the admission time $(p=0.018)$. The results did not show significant differences in mortality rate $(2.5 \%$ vs $10 \% ; p=0.166)$ and LOS $(p=0.866)$ between patients in the finasteride and the control group.

Conclusion: A short course of finasteride administration partially improves $\mathrm{O}_{2}$ saturation but does not influence other outcomes in hospitalized male patients aged $\geq 50$ years with COVID-19 pneumonia. Further research in a large scale with longer follow-up is required to help clarify the role of finasteride in this setting.

Keywords: Finasteride, Adult, Male, Therapy, COVID-19 Infection

Conflicts of Interest: None declared

Funding: Qazvin University of Medical Sciences (project number: 14004290).

\section{${ }^{*}$ This work has been published under CC BY-NC-SA 1.0 license. \\ Copyright $\oplus$ Iran University of Medical Sciences}

Cite this article as: Zarehoseinzade E, Allami A, Ahmadi M, Bijani B, Mohammadi N. Finasteride in hospitalized adult males with COVID-19: A risk factor for severity of the disease or an adjunct treatment: A randomized controlled clinical trial. Med J Islam Repub Iran. 2021 (3 Mar);35:30. https://doi.org/10.47176/mjiri.35.30

\section{Introduction}

In 2020, COVID-19 pneumonia has become a leading cause of morbidity and mortality in many countries

Corresponding author: Dr Abbas Allami, allami9@yahoo.com

1. Department of Infectious Diseases, Clinical Research Development Unit, BouAli Sina Hospital, Qazvin University of Medical of Sciences, Qazvin, Iran

2. Qazvin Blood Transfusion Organization, Qazvin, Iran

3. Children Growth Research Center, Research Institute for Prevention of NonCommunicable Diseases, Qazvin University of Medical Sciences, Qazvin, Iran

4. Canada Optimax Access Consultation, Ottawa, Canada worldwide, particularly among the elderly (1). Since COVID-19 was first reported, a worldwide pandemic has

$\uparrow$ What is "already known" in this topic:

There is controversy about the efficacy of $5 \alpha$-reductase inhibitors in COVID-19 patients. Some assumed they might be a risk factor for deterioration and others proposed it as a possible adjunct treatment for moderate to severe COVID-19 infection in the elderly.

$\rightarrow$ What this article adds:

This study is the first and only interventional research on COVID-19 pneumonia outcome in hospitalized male patients aged $\geq 50$ years. A short course of finasteride administration partially improves peripheral capillary $\mathrm{O}_{2}$ saturation. 
ensued affecting more than 24000000 people as of September 2020. Only oxygen and other supportive care may help improve outcomes in patients with COVID-19 pneumonia and rare pro gress has been made in the treatment of COVID patients (2).

During the Covid-19 pandemic, epidemiological reports unveiled a disproportionate high rate of severe cases and disease outcomes among adult males compared to adult females (with short-term follow-up, males have 65\% higher mortality). Also, convalescence from COVID-19 among the elderly takes longer, and complications and death are also more frequent than in younger adults $(3,4)$.

Research to date has not yet clearly determined the reason for differences of COVID-19 infection severity and outcome between adult females and males. This issue was discussed theoretically in 4 earlier studies (5-8). These studies have introduced the hypothesis that finasteride has a beneficial or harm effect on clinical outcomes in adults with COVID-19 pneumonia. Hoffmann et al propose that the lower rate of severe COVID-19 infection in female patients may be attributed to the molecular mechanism required for SARS-CoV-2 infectivity (ie, lower androgen receptor (AR) expression in females). SARS-CoV-2 cell entry depends on priming of a viral spike surface protein by transmembrane protease serine 2 (TMPRSS2) present in type II pneumocytes (9). TMPRSS2 expression is associated with an increase in AR expression, specifically connecting AR expression to SARSCoV-2 due to ARregulated TMPRSS2 gene promoter $(7,10)$. Angiotensinconverting enzyme 2 (ACE2) is the attachment molecule to the viral spike surface protein "receptor of SARS-CoV2". ACE2 activity has been shown to be reduced by the decrease of androgen hormones, possibly due to decreased expression of ACE2 (6). The US Food and Drug Administration (FDA) approved that 5-alpha reductase inhibitor (finasteride) demonstrated reduction of activation of AR in multiple tissues.

In another paper, authors assumed finasteride might increase androgen concentration in lungs hampering their regeneration. It might result in impairment of spontaneous regeneration capacity and prolonged or deteriorated recovery prognosis. According to the presented hypothesis, patients receiving 5-alpha-reductase inhibitors (5-ARIs) might be vulnerable to COVID-19 infection with poorer prognosis (5).

However, no observational or interventional studies on the effect of finasteride on the treatment of COVID pneumonia was found in our literature review. Taken together, the evidence warrants further studies to elucidate the role, if any, of the AR on the severity of COVID-19 infection. The study aim was to assess the influence of adjunctive treatment with finasteride on the outcomes of hospitalized adult male patients with COVID-19 pneumonia.

\section{Methods}

\section{Study Design}

We conducted a clinical randomized controlled trial in hospitalized male patients aged 50 years or older, who are predisposed to higher AR expression and may also be suffering from benign prostatic hyperplasia (BPH), with
COVID-19 pneumonia. Patients were prospectively enrolled between May and June 2020 at BouAli Sina hospital (a tertiary referral center during the COVID-19 outbreak), Qazvin, Iran. Patients were considered eligible if they met the following criteria: (1) provided informed consent; (2) had clinical symptoms suggestive of COVID19 pneumonia, including cough (with or without sputum), fever, pleuritic chest pain, or dyspnea; (3) chest computed tomography (CT) scan findings compatible with COVID19 or positive real time reverse transcription polymerase chain reaction RT-PCR of COVID-19; (4) male patients aged 50 years and older; and (5) moderate and sever disease.

Patients were excluded from the study if one of the following criteria applied: the presence of severe immunosuppression (eg, use of immunosuppressants); malignancy; any likely infection other than COVID-19 pneumonia; and indications that the patient was unable and/or unlikely to comprehend and/or follow the protocol; liver function abnormalities (as finasteride is metabolized extensively in the liver); and a positive drug history of finasteride medication or hypersensitivity to any component of this medication. If there was any violation of the protocol, the patient was excluded from the final analysis.

We calculated that 40 patients were needed in both groups to detect a mean difference of 2 days LOS $(5 \pm 1.7$ vs $7 \pm 1.7$ days) between finasteride and control groups, with a power of $80 \%$ and an alpha level of 0.05 (by statistics and sample size calculator). Formula:

$$
n \geq \frac{\left(\mathrm{Z}_{1-\frac{\alpha}{2}}+\mathrm{Z}_{1-\beta}\right)^{2}\left(\sigma_{1}^{2}+\frac{\sigma_{2}^{2}}{r}\right)}{\left(\mu_{1}-\mu_{2}\right)^{2}}
$$

Patients were randomly allocated into 2 therapeutic groups in a 1:1 ratio to receive either only common care based on "Iranian Guideline of Hospitalized COVID-19 Patients' Management (V 5)" or common care plus finasteride (as adjuvant). The randomization sequence was generated using Statistics and Sample Size application version 1.0. A simple randomized list was produced for a sample size of 80 and the participants were placed into 2 groups of case and control with numeric sequential unique identifiers (simple or unrestricted randomization). Forty patients in case group received a film coated tablet containing $5 \mathrm{mg}$ finasteride (Aburaihan pharmaceutical Co, Iran) once daily for 7 days.

This study was a partial double-blind study. During the treatment phase, the investigators could ascertain the patients' study-drug assignment (only in the event of an emergency). During the study, to minimize possible sources of bias (ie, report more favorable outcomes or even reporting subjective efficacy endpoints or adverse effects in patients with previous experience of finasteride and reporting treatment responses or adverse events by the observer), patients and health care professionals who were undertaking the outcome assessment of the primary outcome were blinded to the group to which the subject was 
assigned.

Clinical data were measured at enrolment. Hypertension was defined as an average systolic blood pressure (SBP) greater than or equal to $140 \mathrm{mmHg}$ or an average diastolic blood pressure (DBP) greater than or equal to $90 \mathrm{mmHg}$, or currently using blood pressure (BP)-lowering medication. A generalized formula of the mean arterial pressure (MAP) is as follows:

$\mathrm{MAP}=\mathrm{DP}+0.01 \times \exp (4.14-40.74 / \mathrm{HR})(\mathrm{SP}-\mathrm{DP})$, where HR is the heart rate (11).

All patients were treated according to "Iranian Guideline of Hospitalized COVID-19 Patients Management (version 5)". This comprised a heparin prophylaxis in combination with a supplemental oxygen and intravenous or oral fluid therapy. To avoid exaggerated estimates of treatment effect, baseline and follow-up clinical and paraclinical data measurement and recording were performed by nurses with adequate experience. The investigators did not influence decisions concerning discharge.

The primary endpoint was death/alive status and length of hospital stay (LOS) and secondary endpoints were peripheral capillary oxygen saturation, respiratory rate, and inflammatory markers changes. A CURB-65 severity score was calculated, and 1 point was given for each feature present (range, $0-4$ points) $(12,13)$. All patients were observed and subsequently asked about adverse events. Renal function assessment was performed on presentation.

Assessment of gas exchange requires knowledge of fractional inspired oxygen tension $\left(\mathrm{FiO}_{2}\right)$ unless the patient is breathing room air. Hence, all peripheral capillary oxygen saturation was measured in breathing room air at rest.

This trial was registered with the Iranian Registry of Clinical Trials website (registration ID IRCT20200505047318N1). The study protocol was approved by the medical ethics committee of Qazvin University of Medical Sciences (registration ID IR.QUMS.REC.1399.080). All participants were provided with information about the study's purpose and gave informed consent to participate in the study, according to the principles of medical ethics of the World Health Organization and the seventh revision of the Declaration of Helsinki 2008.

\section{Statistical Analysis}

The data were summarized as frequencies or percentages for categorical variables and as medians and interquartile ranges (IQR) (all the variable distributions were skewed). We compared continuous variables using the Mann-Whitney test and proportions using the $\chi^{2}$ test or Fisher's exact test. Moderation analyses were conducted using the SPSS macro-PROCESS V 3.5. The PROCESS macro produces bootstrapped unstandardized regression output as well as estimates of the effect of the focal predictor variables (LOS and death/alive status) at values of the moderator variables (ie, diabetes melitus) (14). $\mathrm{P} \leq 0.05$ was considered statistically significant. The Statistical Package for Social Sciences software, version 25.0 (SPSS $^{\circledR}$, Armonk, NY, USA) was used for data management and statistical analysis.

\section{Results}

In this study, of the 90 hospitalized male patients diagnosed with COVID-19 pneumonia who met the inclusion criteria, 8 were unwilling to continue the study. The remaining 82 patients were randomized, and 2 patients, one in each group, were excluded due to a positive drug history of finasteride. Data from the final 80 randomized patients were analyzed (Fig. 1). Demographic Information is presented in Table 1.

Patients were evenly distributed in the 2 groups, except those with diabetes mellites history; (5 [12.5\%] vs 15 $[37.5 \%]$ patients; $p=0.020)$. Other clinical and paraclinical features, such as Glasgow coma scale, peripheral capillary oxygen saturation, white blood cell and platelet count, and erythrocyte sedimentation rate, were not significantly different between the 2 groups (Tiable 1).

The results did not show significant differences in mortality rate $(1[2.5 \%]$ vs $4[10.0 \%] ; p=0.166)$ and LOS $(\mathrm{p}=0.866)$ between patients in the finasteride and the control groups (Table 2).

We found a significant difference in peripheral capillary $\mathrm{O}_{2}$ saturation on the fifth day among the 2 study groups $(\mathrm{p}=0.016)$ (Fig. 2).

The results of the binary logistic regression analysis and linear regression analysis showed diabetes is not a significant predictor of the outcome variables (for death/alive status [model 1 Hayes: $\mathrm{Y}=$ death/alive status, $\mathrm{X}=$ group and $\mathrm{W}=\mathrm{DM} ; \mathrm{p}=0.989$ ] and for LOS [model 1 Hayes: $\mathrm{Y}=$ LOS, $\mathrm{X}=$ group and $\mathrm{W}=\mathrm{DM} ; \mathrm{p}=0.398]$ ).

Two patients developed hospital-acquired pneumonia and were treated in the intensive care unit (one patient in each group). Other patients in the finasteride group and in the control group did not have any treatment-related adverse events.

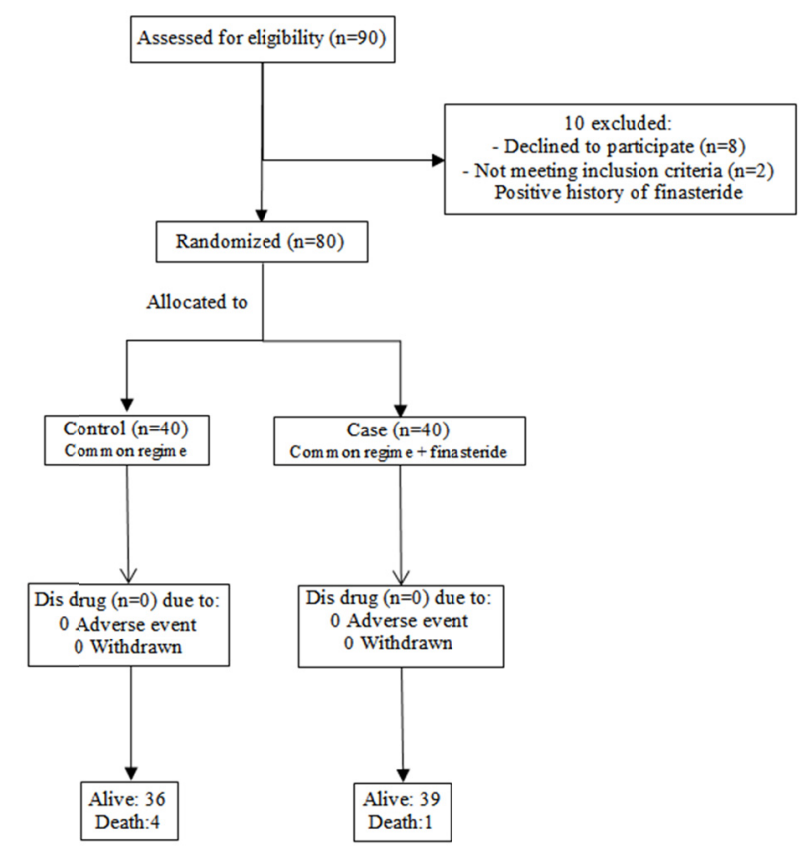

Fig. 1. Design Trial 
Table 1. Baseline Characteristics and Severity Score in the Study Groups

\begin{tabular}{|c|c|c|c|c|c|}
\hline \multirow{2}{*}{ Characteristics } & & \multicolumn{2}{|c|}{ Group } & \multirow[b]{2}{*}{ Total $(n=80)$} & \multirow[b]{2}{*}{$\mathrm{p}$} \\
\hline & & $\begin{array}{l}\text { Finasteride }+ \text { Common } \\
\text { regimen }(n=40)\end{array}$ & $\begin{array}{l}\text { Common regimen } \\
(\mathrm{n}=40)\end{array}$ & & \\
\hline \multicolumn{2}{|l|}{ Age (year) } & $71[62-81]$ & $72[65-77]$ & $72[64-78]$ & 0.904 \\
\hline \multicolumn{2}{|l|}{ Diabetes mellitus (\%) } & $5(12.5)$ & $15(37.5)$ & $20(25.0)$ & $0.020^{*}$ \\
\hline \multicolumn{2}{|l|}{ Hypertension (\%) } & $24(60.0)$ & $29(72.5)$ & $53(66.3)$ & 0.344 \\
\hline \multicolumn{2}{|c|}{ Cardiac disease $(\%)$} & $17(42.5)$ & $14(35.0)$ & $31(38.8)$ & 0.646 \\
\hline \multicolumn{2}{|c|}{ Chronic obstructive pulmonary disease } & $3(7.5)$ & $7(17.5)$ & $10(12.5)$ & 0.310 \\
\hline \multicolumn{2}{|c|}{ Respiratory rate (breath/min) } & $18[18-20]$ & $19[18-20]$ & $19[18-20]$ & 0.189 \\
\hline \multicolumn{2}{|c|}{ Systolic blood pressure (mmHg) } & $120[120-135]$ & $130[120-140]$ & $128[120-140]$ & 0.265 \\
\hline \multicolumn{2}{|c|}{ Diastolic blood pressure (mmHg) } & $80[70-80]$ & $80[70-90]$ & $80[70-90]$ & 0.426 \\
\hline \multicolumn{2}{|c|}{ Mean Arterial Pressure (mmHg) } & $96[92-103]$ & 97 [91- 109] & $96[91-106]$ & 0.453 \\
\hline \multicolumn{2}{|c|}{ Pulse rate (beats/min) } & $90[78-100]$ & $85[81-90]$ & $88[80-95]$ & 0.205 \\
\hline \multicolumn{2}{|c|}{ Temperature $\left({ }^{\circ} \mathrm{C}\right)$} & $36.8[36.5-37.8]$ & $36.9[36.5-37.2]$ & $36.8[36.5-37.4]$ & 0.877 \\
\hline \multicolumn{2}{|c|}{ Peripheral capillary $\mathrm{O}_{2}$ saturation } & $90[84-92]$ & 89 [86-92] & $90[85-92]$ & 0.806 \\
\hline \multirow[t]{4}{*}{ Glasgow Coma Scale (G } & $\leq 10$ & $3(7.5)$ & $0(0.0)$ & $3(3.9)$ & 0.136 \\
\hline & $11-12$ & $3(7.5)$ & $1(2.5)$ & $4(5.1)$ & \\
\hline & $13-14$ & $4(10.0)$ & $8(20.0)$ & $12(15.0)$ & \\
\hline & 15 & $30(75.0)$ & $31(77.5)$ & $61(76.3)$ & \\
\hline \multicolumn{2}{|c|}{ White blood cells (per mL) } & $8.0[4.6-11.4]$ & $8.9[6.8-11.7]$ & $8.4[6.3-11.6]$ & 0.187 \\
\hline \multicolumn{2}{|c|}{ Absolute Lymphocyte count per $\mu \mathrm{l}$ ) } & $1063[797-1441]$ & $1144[851-1455]$ & $1116[821-1455]$ & 0.498 \\
\hline \multicolumn{2}{|c|}{ Platelets count (per ml) } & 159 [117-234] & 183 [134- 211] & $162[130-223]$ & 0.557 \\
\hline \multicolumn{2}{|c|}{ Erythrocyte sedimentation rate $(\mathrm{mm} / \mathrm{h})$} & $29[9-45]$ & $28[15-44]$ & $28[14-44]$ & 0.952 \\
\hline \multicolumn{2}{|c|}{ C-Reactive Protein (mg/dL) } & $23[10-40]$ & $22[6-43]$ & $23[7-42]$ & 0.704 \\
\hline \multicolumn{2}{|c|}{ Blood Urea Nitrogen (mg/dL) } & $22[17-29]$ & 23 [19- 29] & $22[18-29]$ & 0.512 \\
\hline \multicolumn{2}{|c|}{ Creatinine $(\mathrm{mg} / \mathrm{dL})$} & $1.0[0.9-1.3]$ & $1.1[0.9-1.3]$ & $1.1[0.9-1.3]$ & 0.615 \\
\hline \multirow[t]{4}{*}{ Risk class (CURB-65) } & 0 & $7(17.5)$ & $1(2.5)$ & $8(10.0)$ & 0.443 \\
\hline & 1 & $12(30.0)$ & $16(40.0)$ & $28(35.0)$ & \\
\hline & 2 & $18(45.0)$ & $23(57.5)$ & $41(51.2)$ & \\
\hline & 3 & $3(7.5)$ & $0(0.0)$ & $3(3.8)$ & \\
\hline
\end{tabular}

$\mathrm{n}(\%)$ or median [IQR]; IQR = Interquartile Range; CURB-65 = Mental Confusion; Urea $>20 \mathrm{mg} / \mathrm{dL}$; Respiratory Rate $\geq 30 /$ min; Low Blood Pressure (diastolic blood pressure $\leq 60 \mathrm{~mm} \mathrm{Hg}$ or systolic blood pressure $>90 \mathrm{~mm} \mathrm{Hg}$ ); Age $\geq 65$ years; COVID-19 Pneumonia.

Table 2. Length of Hospital Stay, Outcome and Parameters of Disease Severity in Fifth Day Admission Among Male Adults With COVID-19 Pneumonia.

\begin{tabular}{|c|c|c|c|c|}
\hline \multirow{2}{*}{ Characteristics } & \multicolumn{4}{|c|}{$\begin{array}{c}\text { Group } \\
\mathrm{n}(\%) \text { or median }[\mathrm{IQR}] \\
\end{array}$} \\
\hline & & Finasteride Plus Common regime $(n=40)$ & Common Regimen $(n=40)$ & $\mathrm{p}$ \\
\hline \multicolumn{2}{|l|}{ Respiratory rate (breath/min) } & $18[17-18]$ & $18[17-18]$ & $0.940 *$ \\
\hline \multicolumn{2}{|c|}{ Systolic blood pressure (mmHg) } & $115[103-120]$ & $120[110-130]$ & $0.090 *$ \\
\hline \multicolumn{2}{|c|}{ Diastolic blood pressure (mmHg) } & $70[70-80]$ & $80[70-80]$ & $0.074 *$ \\
\hline \multicolumn{2}{|c|}{ Pulse rate (beats/min) } & $79[69-84]$ & $73[66-80]$ & $0.126^{*}$ \\
\hline \multicolumn{2}{|c|}{ Temperature $\left({ }^{\circ} \mathrm{C}\right)$} & $36.7[36.5-36.8]$ & $36.7[36.5-36.9]$ & $0.731 *$ \\
\hline \multicolumn{2}{|c|}{ Peripheral capillary $\mathrm{O}_{2}$ saturation } & $92[89-94]$ & $89[86-92]$ & $0.016^{* \dagger}$ \\
\hline \multicolumn{2}{|c|}{ White blood cells (per $\mu \mathrm{l})$} & $7100[5000-9900]$ & $6900[5900-8950]$ & $0.773 *$ \\
\hline \multicolumn{2}{|c|}{ Absolute lymphocyte count (per $\mu \mathrm{l})$} & $1057[630-1491]$ & $1312[1062-1440]$ & $0.229 *$ \\
\hline \multicolumn{2}{|c|}{ Platelets count (per $\mu \mathrm{l})$} & $200[165-254]$ & $195[157-237]$ & $0.625^{*}$ \\
\hline \multicolumn{2}{|c|}{ Erythrocyte sedimentation rate $(\mathrm{mm} / \mathrm{h})$} & $29[13-40]$ & $28[20-44]$ & $0.676^{*}$ \\
\hline \multicolumn{2}{|c|}{ C-Reactive Protein $(\mathrm{mg} / \mathrm{dL})$} & $18[5-50]$ & $28[20-40]$ & $0.255^{*}$ \\
\hline \multicolumn{2}{|l|}{ Blood Urea Nitrogen $(\mathrm{mg} / \mathrm{dL})$} & $18[15-31]$ & $21[15-29]$ & $0.602 *$ \\
\hline \multicolumn{2}{|l|}{ Creatinine $(\mathrm{mg} / \mathrm{dL})$} & $1[1-1]$ & $1[1-1]$ & $0.670 *$ \\
\hline \multicolumn{2}{|l|}{ Length of hospital stay (day) } & $10[6-16]$ & $10[6-14]$ & $0.866^{*}$ \\
\hline \multicolumn{2}{|l|}{ Length of ICU stay (day) } & $0[0-0]$ & $0[0-0]$ & $0.902 *$ \\
\hline \multicolumn{2}{|l|}{ Length of intubation (day) } & $0[0-0]$ & $0[0-0]$ & $0.539 *$ \\
\hline \multirow[t]{3}{*}{ Glasgow Coma Scale (GCS) } & $\leq 8$ & $2(5)$ & $2(5)$ & $0.730 * *$ \\
\hline & $\overline{9}$ to 14 & $8(17.5)$ & $7(17.5)$ & \\
\hline & 15 & $31(77.5)$ & $31(77.5)$ & \\
\hline \multirow[t]{2}{*}{ Outcome } & discharge & $39(97.5)$ & $36(90)$ & $0.166^{* * *}$ \\
\hline & expire & $1(2.5)$ & $4(10)$ & \\
\hline
\end{tabular}

\section{Discussion}

To the best of our knowledge, this clinical trial study was the first to examine the effects of finasteride as adjunctive therapy on outcome, hypoxia, and inflammatory biomarkers in hospitalized adult male patients with COVID-19 by assessing clinical and paraclinical parameters. This study may help clinicians to optimize the
COVID infection management to decrease its mortality and morbidity.

Only patient peripheral capillary $\mathrm{O}_{2}$ saturation on the fifth day was significantly higher in the finasteride compared with the control group. Finasteride could reduce hypoxia-inducible factor-1alpha (HIF-1 $\alpha$ ), which reduces vascular endothelial growth factor (VEGF), which in turn 


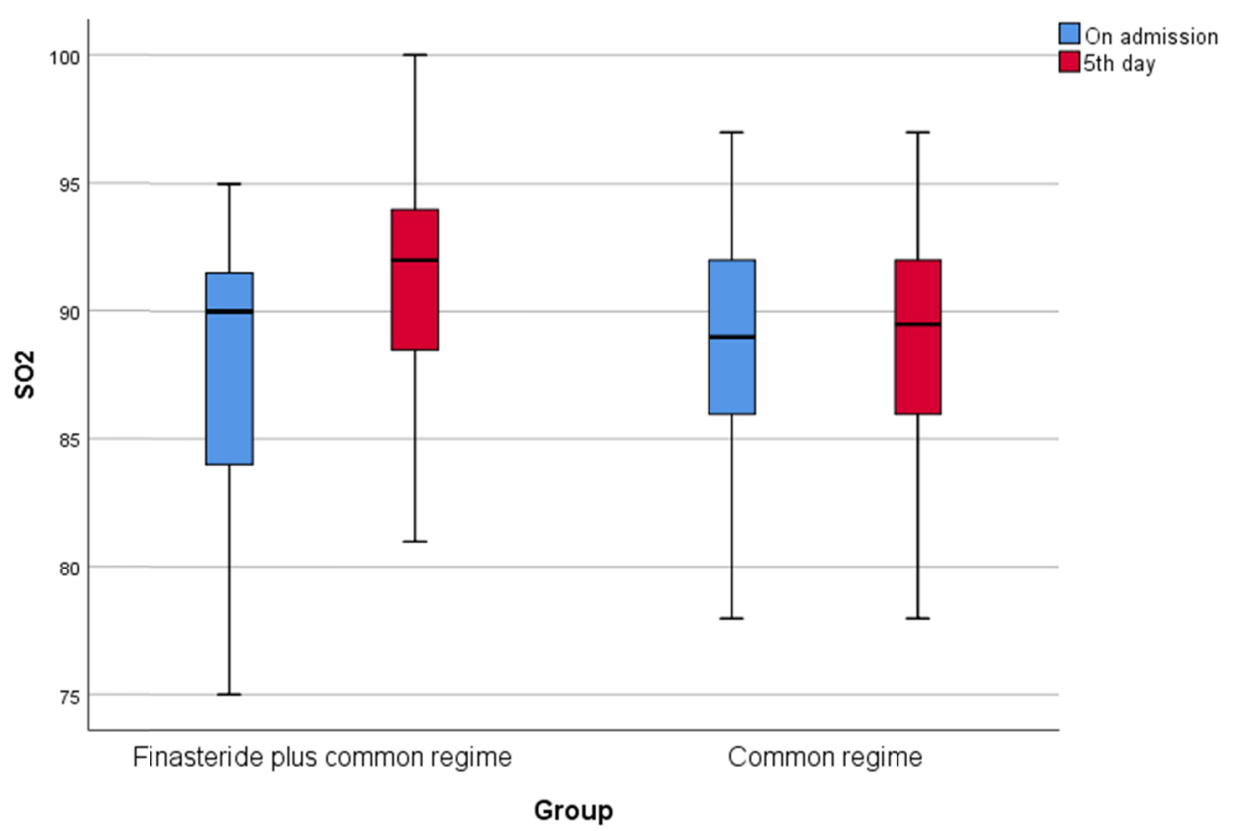

Fig. 2. Box Plot of the peripheral capillary $\mathrm{O}_{2}$ saturation trends during hospital admission for the 2 study groups

reduces micro vessel density (MVD). Finasteride administration in BPH results in a statistically significant suppression of hypoxia marker in BPH (HIF-1 $\alpha$ expression) (15).

Although a trend towards improved clinical outcomes was observed in the finasteride group, these differences were not statistically significant at day 5 . Reduction in LOS is an important goal in the treatment of patients with COVID-19 pneumonia. In our study, the finasteride group patients had not shorter LOS than control patients.

One hypothetical study assumed finasteride might be a risk factor for deterioration of COVID-19 pneumonia in the elderly (5) and 2 others proposed finasteride administration as a possible adjunctive treatment $(6,16)$. Based on our result, a short course administration of finasteride does not seem to deteriorate outcomes of COVID-19 pneumonia in male adults but even improves peripheral capillary $\mathrm{O}_{2}$ saturation. Two recent reports are compatible with our study. In a recent retrospective cohort analysis on male participants with laboratory confirmed SARS-CoV-2 infection, it was demonstrated that males using the 5-ARIs display drastically reduced symptoms of COVID-19 disease in an outpatient setting (17). Another prospective cohort study was conducted from the data of men hospitalized due to COVID-19. The participants were categorized into 2 cohorts: those taking antiandrogens for at least 6 months or those not taking antiandrogens prior to hospitalization. The participants were followed for a period of 60 days from the date of hospitalization. The relative risk for ICU admission for those taking antiandrogens compared with the age-matched group was RR 0.19 (16).

Adverse events related to treatment with finasteride in our study were low and did not differ from the control group. Adverse effects such as decrease in sexual and genitourinary complications were reported with long-term finasteride consumption; however, at recommended dos- ages and duration, finasteride is well-tolerated. Finasteride is generally well tolerated; adverse reactions are usually mild and transient (18).

There are a number of limitations in the present study. A limitation of the present study was that the finasteride and dihydrotestosterone levels in our study population was not directly assessed. The lack of a placebo group necessitated a single-blind design, which may have influenced the study outcomes. To prevent bias in parameter estimates, health care professionals who were undertaking primary outcome assessment did not have a priori knowledge of group assignment (ie, partially blinded). In addition to, patients took numerous medications. Also, the follow-up period was short and in conditions such as COVID-19, much longer periods are warranted to evaluate lasting treatment effects. Furthermore, the follow-up period lacked a control group because of funding constraints. Additionally, although patients were usually discharged when they reached the discharge criteria listed in the national COVID-19 guidelines, various comorbid disease of patients and their socioeconomic status might influence the decision-making of physicians.

\section{Conclusion}

A short course of finasteride administration partially improves peripheral capillary $\mathrm{O}_{2}$ saturation but does not influence other outcomes in hospitalized male patients aged 50 years and older with COVID-19 pneumonia. Further research in a large scale with longer follow-up is required to help clarify the role of finasteride in the treatment of COVID-19 pneumonia. Until then, we should be cautious and not recommend routine administration of finasteride for COVID-19 treatment.

\section{Acknowledgements}

The authors would like to thank the medical and nursing 
staff at the BouAli Sina Medical Centre who participated in the study.

\section{Conflict of Interests}

The authors declare that they have no competing interests.

\section{References}

1. Temgoua MN, Kuate LM, Ngatchou W, Sibetcheu A, Toupendi ZN, Belobo G, et al. COVID-19 pandemic: do we need systematic screening of patients with cardiovascular risk factors in Low and Middle-Income Countries (LMICs) for preventing death? Pan Afr Med J. 2020;35(Suppl 2):11.

2. Mehta P, McAuley DF, Brown M, Sanchez E, Tattersall RS, Manson JJ, et al. COVID-19: consider cytokine storm syndromes and immunosuppression. Lancet (London, England). 2020;395(10229):1033-1034.

3. Li LQ, Huang T, Wang YQ, Wang ZP, Liang Y, Huang TB, et al. COVID-19 patients' clinical characteristics, discharge rate, and fatality rate of meta-analysis. J Med Virol. 2020;92(6):577-583.

4. Guan WJ, Ni ZY, Hu Y, Liang WH, Ou CQ, He JX, et al. Clinical characteristics of coronavirus disease 2019 in China. N Engl J Med. 2020;382(18):1708-1720.

5. Adamowicz J, Juszczak K, Drewa T. May patients receiving 5-alphareductase inhibitors be in higher risk of COVID-19 complications? Med Hypothes. 2020;40:109751.

6. Goren A, McCoy J, Wambier CG, Vano-Galvan S, Shapiro J, Dhurat $\mathrm{R}$, et al. What does androgenetic alopecia have to do with COVID-19? An insight into a potential new therapy. Dermatol Ther. 2020:e13365.

7. Wambier CG, Goren A, Vaño-Galván S, Ramos PM, Ossimetha A, Nau G, et al. Androgen sensitivity gateway to COVID-19 disease severity. Drug Dev Res. 2020;81(7):771-776.

8. Kroumpouzos G. Effects of 5-alpha reductase inhibitors on lung function: A reason for discontinuation during COVID-19 pandemic? Dermatol Ther. 2020;33(4):e13535.

9. Hoffmann M, Kleine-Weber H, Schroeder S, Krüger N, Herrler T, Erichsen S, et al. SARS-CoV-2 cell entry depends on ACE2 and TMPRSS2 and is blocked by a clinically proven protease inhibitor. Cell. 2020;181(2):271-280.e278.

10. Mikkonen L, Pihlajamaa P, Sahu B, Zhang FP, Jänne OA. Androgen receptor and androgen-dependent gene expression in lung. Mol Cell Endocrinol. 2010;317(1-2):14-24

11. Moran D, Epstein Y, Keren G, Laor A, Sherez J, Shapiro Y. Calculation of mean arterial pressure during exercise as a function of heart rate. Appl Human Sci. 1995;14(6):293-295.

12. Jones BE, Jones J, Bewick T, Lim WS, Aronsky D, Brown SM, et al. CURB-65 pneumonia severity assessment adapted for electronic decision support. Chest. 2011;140(1):156-163.

13. Sharafi S, Allami A. Efficacy of zinc sulphate on in-hospital outcome of community-acquired pneumonia in people aged 50 years and over. Int J Tuberc Lung Dis. 2016;20(5):685-688.

14. Hayes AF. Partial, conditional, and moderated moderated mediation: Quantification, inference, and interpretation. Commun Monogr. 2018;85(1):4-40.

15. Chen Y, Xu H, Shi Q, Gu M, Wan X, Chen Q, et al. Hypoxiainducible factor $1 \alpha$ (HIF-1 $\alpha$ ) mediates the epithelial-mesenchymal transition in benign prostatic hyperplasia. Int $\mathrm{J}$ Clin Exp Pathol. 2019;12(1):295-304.

16. Goren A, Wambier CG, Herrera S, McCoy J, Vaño-Galván S, Gioia $\mathrm{F}$, et al. Anti-androgens may protect against severe COVID-19 outcomes: results from a prospective cohort study of 77 hospitalized men. J Eur Acad Dermatol Venereol. 2020.

17. McCoy J, Cadegiani FA, Wambier CG, Herrera S, Vaño-Galván S, Mesinkovska NA, et al. 5-Alpha-Reductase Inhibitors are Associated with Reduced Frequency of COVID-19 Symptoms in Males with Androgenetic Alopecia. J Eur Acad Dermatol Venereol. 2020.

18. Hirshburg JM, Kelsey PA, Therrien CA, Gavino AC, Reichenberg JS. Adverse effects and safety of 5-alpha reductase inhibitors (finasteride, dutasteride): a systematic review. J Clin Aesthet Dermatol. 2016;9(7):56-62. 